Proceedings of the 2005 Winter Simulation Conference

M. E. Kuhl, N. M. Steiger, F. B. Armstrong, and J. A. Joines, eds.

\title{
A STUDY OF VARIANCE REDUCTION TECHNIQUES FOR AMERICAN OPTION PRICING
}

\author{
Christiane Lemieux \\ Jennie La \\ Department of Mathematics and Statistics \\ University of Calgary \\ 2500 University Drive N.W. \\ Calgary, AB, T2N 1N4, CANADA
}

\begin{abstract}
American option pricing is a challenging problem in financial mathematics for which several approaches have been proposed in the last few years. In this paper, we consider the regression-based method of Longstaff and Schwartz (2001) to price these options, and then investigate the use of different variance reduction techniques to improve the efficiency of the Monte Carlo estimators thus obtained. The techniques considered have been shown to work well for European option pricing. One of them is importance sampling, in which the approach of Glasserman, Heidelberger, and Shahabuddin (1999) is applied to find an appropriate change of measure. We also consider control variates and randomized quasi-Monte Carlo methods, and use numerical experiments on American Asian call options to investigate the performance of these methods.
\end{abstract}

\section{INTRODUCTION}

Option pricing is an important problem in financial mathematics, which continues to challenge researchers and practitioners due to the increased complexity in models and options considered. For European options - options that can only be exercised at the expiration time $T-$ it is often possible to derive closed-form solutions for their value at time $t<T$. When this is not possible, different approaches can be used to get accurate approximations. Among these approaches, those based on Monte Carlo simulations combined with variance reduction techniques are often quite competitive: see Broadie and Glasserman (1997), Glasserman (2004) and the references therein for examples. For American options - options that can be exercised before the expiration time - the problem is more challenging since in that case, it involves finding the optimal exercise time in addition to the estimation of the option's value at a given time. More precisely, for a payoff at time $t$ given by $C(t, \mathbf{S}(\cdot))$, where $\mathbf{S}(\cdot)$ is the vector of underlying assets upon which the option depends, a risk-free interest rate of $r$, and a sequence of $b$ possible exercise times given by $t_{1}, \ldots, t_{b}$, the goal here is to evaluate

$$
\mu=\max _{1 \leq j \leq b} \mathrm{E}\left(e^{-r t_{j}} C\left(t_{j}, \mathbf{S}(\cdot)\right)\right),
$$

where the expectation is taken under the risk-neutral measure (Duffie 1996). When the option can only be exercised at a finite number of times like this, it is usually called a Bermudan option. We restrict our attention to this type of option in this paper.

Since the determination of the optimal exercise time typically requires a backward procedure of some kind, it was thought for a while that because of the forward nature of Monte Carlo simulation, this method could not be used to evaluate American options. However, this problem can be overcomed and there now exist several Monte Carlo-based approximations for American option pricing. Not surprisingly, most of these approximations incorporate some type of variance reduction technique to improve their efficiency. For instance, Broadie and Glasserman (1997) use a mesh based on Monte Carlo simulations of the underlying assets and use control variates at different levels to improve the approximations obtained; Boyle, Kolkiewicz, and Tan (2003) investigate the same kind of stochastic mesh but use low-discrepancy point sets to drive the simulations and incorporate control variates as well; Avramidis and Hyden (1999) also study this mesh-based approach and show how to improve it using importance sampling. Another interesting approach that makes use of Monte Carlo simulations is the one discussed in Haugh and Kogan (2004). We refer the reader to Chapter 8 of Glasserman (2004) for more on Monte Carlo methods in the context of American option pricing.

In this paper, we focus on the regression-based approach discussed in Longstaff and Schwartz (2001) (henceforth denoted LS), which also uses Monte Carlo simulation to generate paths of the underlying assets, and produces a 


\section{Lemieux and La}

low-biased estimator. More precisely, regression is used to produce function approximations for the continuation value of the option at each possible exercise time, which in turn produce estimates of the optimal exercise time. The paths are then used in a second stage to price the option.

In Lemieux (2004), numerical results are given to illustrate how randomized quasi-Monte Carlo methods can be used to reduce the variance of the estimators obtained in the LS approach. The idea is to use a highly-uniform point set (HUPS) to generate the paths - and we assume the same paths are used for both stages of the LS approach - instead of using i.i.d. paths. More details are given in Section 3, and we refer the reader to L'Ecuyer (2004) for a recent survey of the use of quasi-Monte Carlo methods in finance. The use of quasi-Monte Carlo within LS is studied in Chaudhary (2004) as well.

Our goal in this paper is to investigate the use of other variance reduction techniques that have been applied successfully in the context of European option pricing, study their combinations, and compare their performance. The first technique considered is importance sampling, whose use within the LS approach is studied in Moreni (2003) and in Bolia, Glasserman, and Juneja (2004). Here we incorporate it along the lines described in Moreni (2003). That is, importance sampling is used to modify the probability measure under which the paths are sampled. Since those paths are used in both stages, it means both the optimal exercise time and the option price are estimated using importance sampling. To decide how to change the measure, we use the approach of Glasserman, Heidelberger, and Shahabuddin (1999) that was proposed for path-dependent European options. In addition to importance sampling, we consider the use of control variates based on European option prices.

This paper is organized as follows: in Section 2, we describe the LS approach for American option pricing. The variance reduction techniques used to improve the efficiency of the estimators obtained with the LS approach are described in Section 3. Numerical experiments are reported in Section 4 , and concluding remarks are given in Section 5.

\section{REGRESSION-BASED AMERICAN OPTION PRICING}

The method uses $n$ realization paths $\left\{\mathbf{S}^{i}(t), t=\right.$ $\left.0, t_{1}, \ldots, t_{b} ; i=1, \ldots, n\right\}$ of the underlying assets, where $t_{b}=T$ is the expiration time and $t_{1}, \ldots, t_{b}$ are the exercise dates. Since the payoff $C\left(T, \mathbf{S}_{i}(\cdot)\right)$ for each path can be computed at expiration time $T$, the idea is to proceed backward from $T$ to 0 to estimate the optimal exercise time $t_{i}^{*}$ for each path. This is done as follows: set $t_{i}^{*}=T$, then at time $t=t_{b-1}, t_{b-2}, \ldots, t_{1}$, set $t_{i}^{*}=t$ if $C\left(t, \mathbf{S}^{i}(\cdot)\right)>\hat{F}\left(t, \mathbf{S}^{i}(t)\right)$, where $\hat{F}\left(t, \mathbf{S}^{i}(t)\right)$ is an estimate of the continuation value of the option at time $t$ given $\mathbf{S}^{i}(t)$. This estimate is obtained by constructing an approxima- for $i \leftarrow 1$ to $n$ // loop over paths - initialization $t^{*}(i) \leftarrow T$

for $t \leftarrow t_{b-1}$ downto $1 / /$ go backward in time for $i \leftarrow 1$ to $n$ // loop over paths compute $\hat{\beta}_{0}, \ldots, \hat{\beta}_{M}$ compute $\hat{F}\left(t, \mathbf{S}^{i}(t)\right)=\sum_{l=0}^{M} \hat{\beta}_{l} \psi_{l}\left(\mathbf{S}^{i}(t)\right)$ if $C\left(t, \mathbf{S}_{i}(t)\right)>\hat{F}\left(t, \mathbf{S}^{i}(t)\right)$ then

$t^{*}(i) \leftarrow t / /$ update end if end for end for

Figure 1: Longstaff-Schwartz Approach

tion at each time $t$ based on a finite set of multivariate basis functions $\left\{\psi_{l}(\cdot), l=0,1, \ldots, M\right\}$. The coefficients $\beta_{0}, \ldots, \beta_{M}$ for the approximation are estimated by regression of the actualized payoffs (from time $t_{j}^{*}$, for each path $j$ ) against the current value of the assets over the paths that are in-the-money, that is, such that $C\left(t, \mathbf{S}^{i}(\cdot)\right)>0$. More precisely,

$$
\left(\hat{\beta}_{0}, \ldots, \hat{\beta}_{M}\right)^{T}=\left(\Psi^{T} \Psi\right)^{-1} \Psi^{T}\left(y_{1}, \ldots, y_{n^{*}}\right)^{T}
$$

where $n^{*}$ is the number of paths that are in-the-money at time $t, y_{i}=C\left(t_{i}^{*}, \mathbf{S}^{i}(\cdot)\right)$, and $\Psi_{i, l}=\psi_{l}\left(\mathbf{S}^{i}(t)\right)$ for $i=1, \ldots, n^{*}, l=0, \ldots, M$. Then $\left.\hat{F}\left(t, \mathbf{S}^{i}(t)\right)\right)=$ $\sum_{l=0}^{M} \hat{\beta}_{l} \psi_{l}\left(\mathbf{S}^{i}(t)\right)$.

Once the optimal exercise times are estimated for each path, the option's value is estimated as

$$
\hat{\mu}_{\mathrm{ls}}=\frac{1}{n} \sum_{i=1}^{n} e^{-r t_{i}^{*}} C\left(t_{i}^{*}, \mathbf{S}^{i}(\cdot)\right) .
$$

This estimator is low-biased since it uses for each path an estimate of the optimal exercise time. Also, note that even when the $n$ paths used to construct this estimator are independent, the variables $e^{-r t_{i}^{*}} C\left(t_{i}^{*}, \mathbf{S}^{i}(\cdot)\right)$ are not independent since all $n$ paths are used to estimate each $t_{i}^{*}$. Figure 1 summarizes the LS approach using pseudocode.

To conclude this section, let us say a few words on how a path in the LS approach is generated. Here we assume that there exists an updating function $\phi(\mathbf{u}, \mathbf{S}(t), t, \Delta t)$ that generates a price vector at time $t+\Delta t$ given a value of $\mathbf{S}(t)$ at time $t$. The vector $\mathbf{u} \sim U[0,1)^{d}$ represents the source of randomness, where the value of $d$ depends on the model used. Hence a path $\mathbf{S}\left(t_{1}\right), \ldots, \mathbf{S}\left(t_{b}\right)$ can be generated using a vector $\mathbf{u} \sim U[0,1)^{s}$ with $s=d b$ as its source of randomness, by using $\mathbf{S}\left(t_{j}\right)=\phi\left(\mathbf{u}, \mathbf{S}\left(t_{j-1}\right), t_{j-1}, \Delta t\right)$, with, for instance, $\mathbf{u}=\left(u_{(j-1) d+1}, \ldots, u_{j d}\right)$. For example, in a single-asset model under the Black-Scholes model (i.e., $S(\cdot)$ follows a geometric Brownian motion) - which is what 


\section{Lemieux and La}

is used in Section 4 - we have $d=1$ since we can set

$$
\phi(u, S(t), t, \Delta t)=S(t) e^{\left(r-\sigma^{2} / 2\right) \Delta t+\sigma \sqrt{\Delta t} \Phi^{-1}(u)},
$$

where $\Phi^{-1}$ denotes the inverse of a standard normal CDF. Note that the paths can also be generated using alternative methods. For instance, with RQMC methods it is sometimes useful to generate them in a non-sequential way by making use of the Brownian bridge formula for the underlying Brownian motion. We refer the reader to Chaudhary (2004), Glasserman (2004) and L'Ecuyer (2004) for more on this.

\section{VARIANCE REDUCTION TECHNIQUES}

We now describe the techniques used to reduce the variance of the LS estimator $\hat{\mu}_{\mathrm{ls}}$ based on i.i.d. points $\mathbf{u}_{1}, \ldots, \mathbf{u}_{n} \sim$ $U\left([0,1)^{S}\right)$ to generate the $n$ paths.

\subsection{Randomized Quasi-Monte Carlo}

By using a highly-uniform point set to generate the paths instead of i.i.d. points as in Monte Carlo, we can expect the variance of the resulting estimator to be reduced. More precisely, the idea here is to take a point set $\tilde{P}_{n}$ such that (i) each $\mathbf{u}_{i}$ in $\tilde{P}_{n}$ is uniformly distributed over $[0,1)^{s}$; (ii) $\tilde{P}_{n}$ has the same high uniformity as the original HUPS $P_{n}$. Point sets like this can be obtained by using a deterministic construction yielding $n$ points and then randomizing it appropriately. A simple example of this is to use a shifted Korobov lattice (Korobov 1959), which is based on the deterministic point set

$$
P_{n}=\left\{\frac{i}{n}\left(1, a, a^{2}, \ldots, a^{s-1}\right) \bmod 1, i=0, \ldots, n-1\right\},
$$

and a randomization that shifts each point in $P_{n}$ by a random uniform vector $\mathbf{v} \sim U[0,1)^{s}$. That is,

$$
\tilde{P}_{n}=\left\{\left(\mathbf{u}_{i}+\mathbf{v}\right) \bmod 1, \mathbf{u}_{i} \in P_{n}\right\}
$$

where the modl operation is applied coordinate-wise. Other examples of point sets and randomizations can be found in Owen (1998), L'Ecuyer and Lemieux (2002).

Note that property (i) ensures that a path generated from a point $\tilde{\mathbf{u}}_{i}$ in $\tilde{P}_{n}$ has the same distribution as when Monte Carlo is used, while property (ii) suggests that an estimator based on $\tilde{P}_{n}$ should have a smaller variance than a Monte Carlo estimator based on a random point set. There exist theoretical results providing support for this claim (Owen 1998), but it can also be verified empirically. More precisely, one can generate $m$ i.i.d. copies of the RQMC estimator in order to estimate its variance, and then compare with the Monte Carlo estimator's variance.
In the experiments reported in Section 4, in addition to shifted Korobov lattices, we also use two types of digital nets, which are widely used constructions for HUPS. The first one is a Sobol' net (Sobol' 1967, Bratley and Fox 1988), and the second one is a polynomial Korobov lattice (Lemieux and L'Ecuyer 2003). In both cases, the randomization used is a digital shift in base 2 (L'Ecuyer and Lemieux 2002). This randomization technique is similar to the one used for Korobov lattices, except the random shift $\mathbf{v}$ is added to each point $\mathbf{u}_{i}$ in the net using bitwise addition modulo 2. That is, for a digital net $P_{n}=\left\{\mathbf{u}_{1}, \ldots, \mathbf{u}_{n}\right\}$ in base 2 , let

$$
\tilde{P}_{n}=\left\{\mathbf{u}_{i} \oplus \mathbf{v}, i=1, \ldots, n\right\}
$$

where

$$
\mathbf{u} \oplus \mathbf{v}=\left(u^{1} \oplus v^{1}, \ldots, u^{s} \oplus v^{s}\right),
$$

and for each $j=1, \ldots, s$,

$$
u^{j} \oplus v^{j}=\sum_{l=1}^{\infty}\left(\left(u_{l}^{j}+v_{l}^{j}\right) \bmod 2\right) 2^{-l}
$$

where $u_{l}^{j}, v_{l}^{j} \in\{0,1\}$ are from the binary expansion of $u^{j}$ and $v^{j}$, respectively. That is, $u^{j}=\sum_{l=1}^{\infty} u_{l}^{j} 2^{-l}$ and $v^{j}=\sum_{l=1}^{\infty} v_{l}^{j} 2^{-l}$ (where we assume that $u_{l}^{j}$ and $v_{l}^{j}$ are 0 for infinitely many $l$ ).

\subsection{Importance Sampling}

Importance sampling (IS) is a well-known variance reduction technique that is most useful for rare-event simulation, which in the context of option pricing is relevant for options with values close to 0 . It has been used in that context for European options in Vázquez-Abad and Dufresne (1998); Glasserman, Heidelberger, and Shahabuddin (1999); Su and Fu (2000), among others. Its use in the context of the LS approach is discussed in Moreni (2003) and in Bolia, Glasserman, and Juneja (2004). Here we use the framework discussed in Moreni (2003), and incorporate the approach of Glasserman, Heidelberger, and Shahabuddin (1999) designed for path-dependent European options - to find an appropriate change of measure for generating the paths used in the LS approach. More precisely, let $\mathbf{Z}=\left(Z_{1}, \ldots, Z_{S}\right)$ be a vector of normal random variables such that the discounted payoff of the option at expiration time $T$ can be written as a function $g(\mathbf{Z})$ of these variables. The (European) option price can thus be written as

$$
\mu_{\mathrm{eur}}=\int_{[0,1)^{s}} g(\mathbf{z}) f_{\mathbf{Z}}(\mathbf{z}) d z_{1} \ldots d z_{s}
$$




\section{Lemieux and La}

where $f_{\mathbf{Z}}(\mathbf{z})$ is the density function of $\mathbf{Z}$, which is assumed to be $N\left(\mathbf{0}, I_{S}\right)$, where $I_{S}$ denotes the $s \times s$ identity matrix. For instance, in the case of an Asian call option on a single asset based on an arithmetic average taken at times $t_{j}=j \Delta t$, where $\Delta t=T / s$, we have that

$$
g(\mathbf{Z})=e^{-r T} \max \left(0, \frac{1}{s} \sum_{j=1}^{s} S(0) e^{\left(r-\sigma^{2} / 2\right) \Delta t+\sigma \sqrt{\Delta t} Z_{j}}-K\right)
$$

where $Z_{1}, \ldots, Z_{s}$ are i.i.d. standard normal random variables. With IS, the estimator for $\mu_{\text {eur }}$ becomes

$$
\frac{1}{n} \sum_{i=1}^{n} e^{-r T} g\left(\tilde{\mathbf{z}}_{i}\right) L\left(\tilde{\mathbf{z}}_{i}\right)
$$

where $L(\mathbf{z})$ is the likelihood ratio given by $L(\mathbf{z})=$ $f_{\mathbf{Z}}(\mathbf{z}) / h_{\mathbf{Z}}(\mathbf{z}), h_{\mathbf{Z}}(\mathbf{z})$ is the IS density, and $\tilde{\mathbf{z}_{i}}$ is generated under $h_{\mathbf{Z}}(\cdot)$. In Glasserman, Heidelberger, and Shahabuddin (1999), IS densities of the form $N\left(\mu^{*}, I_{S}\right)$ are considered, and a method to find an approximately optimal vector $\mu^{*}$ is presented. In other words, they show how to choose a new mean vector $\mu^{*}$ for $\mathbf{Z}$ that will (approximately) give the estimator with smallest variance among all possible choices for $\mu^{*}$. Using this $\mu^{*}$ for the corresponding American option means we are presumably even further away from the optimal choice, and as expected, we see in Section 4 that IS applied this way does not work as well as for European options, but can still be quite efficient in some cases.

In the context of American option pricing, the IS estimator is obtained as follows (Moreni 2003):

$$
\frac{1}{n} \sum_{i=1}^{n} e^{-r t_{i}^{*}} g_{t_{i}^{*}}\left(\tilde{\mathbf{z}}_{i}\right) L_{t_{i}^{*}}\left(\tilde{\mathbf{z}}_{i}\right)
$$

where $L_{t_{i}^{*}}\left(\tilde{\mathbf{z}}_{i}\right)$ is the likelihood ratio for the variables in $\tilde{\mathbf{z}}_{i}$ used from time 1 to time $t_{i}^{*}$, and $g_{t_{i}^{*}}\left(\tilde{\mathbf{z}}_{i}\right)$ is the payoff obtained at time $t_{i}^{*}$. In other words, paths are generated under the new measure, the LS approach is applied to find the optimal exercise time for each of these paths, and the estimator is then corrected by multiplying by the appropriate likelihood ratio.

\subsection{Control Variates}

Control variates are another method widely used in simulation, with several success stories in the context of finance. We first quickly recall how this technique works, and then focus on the case where it is used in combination with RQMC methods. In the presentation that follows, we restrict ourselves to the case where only one control variate based on an European option is used, which is what is done in Section 4.
Let $\alpha(t, S(\cdot))$ be the payoff for some European option whose value $\mu_{\alpha}$ at time 0 can be evaluated analytically. We can also estimate $\mu_{\alpha}$ by

$$
\hat{\mu}_{\alpha}=\frac{1}{n} \sum_{i=1}^{n} e^{-r T} \alpha\left(T, \mathbf{S}_{i}(\cdot)\right)
$$

where $\mathbf{S}_{1}(\cdot), \ldots, \mathbf{S}_{n}(\cdot)$ denote the simulated paths of the underlying assets. Note that since we are dealing with a European option, the above estimator does not depend on the estimated optimal exercise times found in the LS approach. by

The CV estimator for the American option is then given

$$
\hat{\mu}_{\mathrm{cv}}=\hat{\mu}_{\mathrm{ls}}+\beta\left(\mu_{\alpha}-\hat{\mu}_{\alpha}\right)
$$

where $\beta$ needs to be estimated.

When the $n$ paths are generated using a (randomized) HUPS, the optimal $\beta$ can be written as (Hickernell, Lemieux, and Owen 2005)

$$
\beta_{\mathrm{rqmc}}=\frac{\operatorname{Cov}\left(\hat{\mu}_{\mathrm{rqmc}, \alpha}, \hat{\mu}_{\mathrm{rqmc}, 1 \mathrm{~s}}\right)}{\operatorname{Var}\left(\hat{\mu}_{\mathrm{rqmc}, \alpha}\right)}
$$

where $\hat{\mu}_{\text {rqme }, \alpha}$ and $\hat{\mu}_{\text {rqme,ls }}$ are the estimators for the American option and $\mu_{\alpha}$, respectively, based on a HUPS. We can then estimate $\beta_{\text {rqmc }}$ by

$$
\hat{\beta}_{\mathrm{rqmc}}=\frac{\sum_{p=1}^{m} \hat{\mu}_{\mathrm{rqmc}, \mathrm{ls}, p} \hat{\mu}_{\mathrm{rqmc}, \alpha, p}-m \bar{\mu}_{\mathrm{rqmc}, \alpha} \bar{\mu}_{\mathrm{rqmc}, \mathrm{ls}}}{\sum_{p=1}^{m} \hat{\mu}_{\mathrm{rqmc}, \alpha, p}^{2}-m \bar{\mu}_{\mathrm{rqmc}, \alpha}^{2}},
$$

where $\hat{\mu}_{\text {rqme }, \alpha, p}$ and $\hat{\mu}_{\mathrm{rqmc}, 1 \mathrm{~s}, p}$ denote the version of the estimators $\hat{\mu}_{\text {rqme, } \alpha}$ and $\hat{\mu}_{\text {rqme, }, 1 s}$ obtained with the $p$ th randomized copy of the HUPS,

$$
\bar{\mu}_{\mathrm{rqmc}, \alpha}=\frac{1}{m} \sum_{p=1}^{m} \hat{\mu}_{\mathrm{rqmc}, \alpha, p}
$$

and

$$
\bar{\mu}_{\mathrm{rqmc}, \mathrm{ls}}=\frac{1}{m} \sum_{p=1}^{m} \hat{\mu}_{\mathrm{rqmc}, \mathrm{ls}, p} .
$$

The rqme-cv estimator is then given by

$$
\hat{\mu}_{\mathrm{rqmc}, \mathrm{cv}}=\frac{1}{m} \sum_{p=1}^{m} \hat{\mu}_{\mathrm{rqmc}, \mathrm{cv}, p}
$$

where

$$
\hat{\mu}_{\mathrm{rqmc}, \mathrm{cv}, p}=\hat{\mu}_{\mathrm{rqmc}, 1 \mathrm{~s}, p}+\hat{\beta}_{\mathrm{rqmc}}\left(\mu_{\alpha}-\hat{\mu}_{\mathrm{rqmc}, \alpha, p}\right) .
$$




\section{Lemieux and La}

\section{NUMERICAL RESULTS}

We now present some numerical results to illustrate the performance of the different combinations of techniques on an Asian American call option whose corresponding average is taken over the $b$ exercise times $t_{1}, \ldots, t_{b}$. For the control variate $(\mathrm{CV})$, we use the corresponding European Asian call on the geometric average (Kemna and Vorst 1990), for which a closed-form solution exists.

The RQMC methods used are a shifted Korobov lattice (Kor), a digitally shifted Sobol' net, and a digitally shifted polynomial Korobov lattice (PKor). In each case, we use $n=4096$ points (so this is the number of paths used in the LS approach), and $m=100$ randomizations. The parameters used are $T=1$ year, $r=0.05, S(0)=50$, with exercise times of the form $t_{j}=j / b, j=1, \ldots, b$, for some value $b$ : in Table $1, b=16$ and $\sigma=0.1$, while in Tables 2 and $3, b=64$ and $\sigma=0.3$. In each table, for each method and exercise price $K$, we give the estimator for the option, with the corresponding standard error below. We provide this information for both the American and European options.

Several observations can be made from these results. First, for in-the-money options, IS gives estimators with a larger (negative) bias than without IS. More precisely, in Table 1, when $K=45$ the estimators with IS are about two cents smaller than the ones without IS, while in Table 2, the difference is about 16, 5 and 1 cents, for $K=45,50$ and 55 , respectively. We believe this is due to the fact that as the difference between the American option's value and the European one - called the early exercise premium - gets larger, the two types of options are more and more different, and thus the change of measure that is optimal for the European option gets further away from the one that would be optimal for the American option. This phenomenon is discussed in Moreni (2003) as well.

A similar argument may be used to explain the performance of the control variate: as the optimal exercise time becomes smaller, the correlation between the American option payoff and the control variate - which uses a payoff at expiration - becomes smaller and thus the control variate becomes less effective. More precisely, for Monte Carlo, the reduction factors in the standard error are about 3,6 and 15 for $K=45,50$ and 55, respectively. The corresponding factors in Table 2 are about 2.5, 4 and 5.5. For the RQMC estimators, $\mathrm{CV}$ does not reduce the standard error significantly for $K=45$, while for $K=55$, reduction factors between 5 and 8 can be observed in Table 1, and of about 2 in Table 2. By comparison, for the European option CV can reduce the standard error by factors as large as 92, as seen in Table 1, when $K=45$ for Monte Carlo. Note that as opposed to what happens for American options, $\mathrm{CV}$ tends to work better for in-the-money options in the European case.
Table 1: $T=1, \sigma=0.1$, and $b=16$

\begin{tabular}{|c|c|c|c|c|}
\hline & $\mathrm{MC}$ & Sobol' & Kor & PKor \\
\hline$K=45$ & \multicolumn{4}{|c|}{ American } \\
\hline \multirow[t]{2}{*}{ naive } & 6.393 & 6.392 & 6.392 & 6.392 \\
\hline & 4.30 e- 3 & $5.77 \mathrm{e}-4$ & $7.21 \mathrm{e}-4$ & $5.04 \mathrm{e}-4$ \\
\hline \multirow[t]{2}{*}{ IS } & 6.377 & 6.377 & 6.378 & 6.377 \\
\hline & $9.67 \mathrm{e}-4$ & $3.23 \mathrm{e}-4$ & 4.18 e- 4 & $3.11 \mathrm{e}-4$ \\
\hline \multirow[t]{2}{*}{$\mathrm{CV}$} & 6.393 & 6.392 & 6.392 & 6.392 \\
\hline & $1.41 \mathrm{e}-3$ & $5.70 \mathrm{e}-4$ & $7.11 \mathrm{e}-4$ & $4.98 \mathrm{e}-4$ \\
\hline \multirow[t]{3}{*}{ IS+CV } & 6.377 & 6.377 & 6.378 & 6.377 \\
\hline & 8.40 e-4 & $3.23 \mathrm{e}-4$ & $3.80 \mathrm{e}-4$ & $3.12 \mathrm{e}-4$ \\
\hline & \multicolumn{4}{|c|}{ European } \\
\hline \multirow[t]{2}{*}{ naive } & 6.055 & 6.055 & 6.055 & 6.055 \\
\hline & 4.69 e-3 & $2.32 \mathrm{e}-4$ & $2.97 \mathrm{e}-4$ & $1.63 \mathrm{e}-4$ \\
\hline \multirow[t]{2}{*}{ IS } & 6.054 & 6.055 & 6.056 & 6.055 \\
\hline & $1.36 \mathrm{e}-3$ & $2.34 \mathrm{e}-4$ & $5.14 \mathrm{e}-4$ & 2.78 e-4 \\
\hline \multirow[t]{2}{*}{$\mathrm{CV}$} & 6.055 & 6.055 & 6.055 & 6.055 \\
\hline & $5.51 \mathrm{e}-5$ & 1.68 e-5 & 1.95 e-5 & $1.01 \mathrm{e}-5$ \\
\hline \multirow[t]{2}{*}{$\mathrm{IS}+\mathrm{CV}$} & 6.055 & 6.055 & 6.055 & 6.055 \\
\hline & $5.10 \mathrm{e}-5$ & $1.44 \mathrm{e}-5$ & $2.07 \mathrm{e}-5$ & $1.53 \mathrm{e}-5$ \\
\hline$K=50$ & \multicolumn{4}{|c|}{ American } \\
\hline \multirow[t]{2}{*}{ naive } & 2.000 & 1.999 & 1.999 & 1.999 \\
\hline & 3.38 e- -3 & $4.87 \mathrm{e}-4$ & 6.70 e- 4 & $4.63 \mathrm{e}-4$ \\
\hline \multirow[t]{2}{*}{ IS } & 1.994 & 1.995 & 1.995 & 1.995 \\
\hline & $1.24 \mathrm{e}-3$ & $4.14 \mathrm{e}-4$ & $6.28 \mathrm{e}-4$ & $4.70 \mathrm{e}-4$ \\
\hline \multirow[t]{2}{*}{$\mathrm{CV}$} & 1.998 & 1.999 & 1.999 & 1.999 \\
\hline & $5.44 \mathrm{e}-4$ & $3.93 \mathrm{e}-4$ & $4.27 \mathrm{e}-4$ & $3.53 \mathrm{e}-4$ \\
\hline \multirow[t]{3}{*}{$\mathrm{IS}+\mathrm{CV}$} & 1.995 & 1.995 & 1.995 & 1.995 \\
\hline & 4.65 e- 4 & 2.70 e- 4 & $3.17 \mathrm{e}-4$ & $2.45 \mathrm{e}-4$ \\
\hline & \multicolumn{4}{|c|}{ European } \\
\hline \multirow[t]{2}{*}{ naive } & 1.921 & 1.920 & 1.919 & 1.919 \\
\hline & 3.49 e- -3 & $3.45 \mathrm{e}-4$ & 6.06 e- 4 & $3.64 \mathrm{e}-4$ \\
\hline \multirow[t]{2}{*}{ IS } & 1.918 & 1.920 & 1.919 & 1.920 \\
\hline & 1.30 e-3 & $4.59 \mathrm{e}-4$ & $6.77 \mathrm{e}-4$ & $5.22 \mathrm{e}-4$ \\
\hline \multirow[t]{2}{*}{$\mathrm{CV}$} & 1.919 & 1.920 & 1.920 & 1.920 \\
\hline & $4.54 \mathrm{e}-5$ & $1.68 \mathrm{e}-5$ & 2.23 e- 5 & $1.65 \mathrm{e}-5$ \\
\hline $\mathrm{IS}+\mathrm{CV}$ & 1.920 & 1.920 & 1.920 & 1.920 \\
\hline & $3.82 \mathrm{e}-5$ & 1.94 e-5 & 2.00 e-5 & $1.81 \mathrm{e}-5$ \\
\hline$K=55$ & & $\mathrm{Am}$ & ican & \\
\hline naive & 0.204 & 0.204 & 0.205 & 0.204 \\
\hline & $1.11 \mathrm{e}-3$ & $3.23 \mathrm{e}-4$ & $5.28 \mathrm{e}-4$ & $3.50 \mathrm{e}-4$ \\
\hline IS & 0.204 & 0.204 & 0.204 & 0.204 \\
\hline & $2.74 \mathrm{e}-4$ & $1.54 \mathrm{e}-4$ & $1.82 \mathrm{e}-4$ & $1.50 \mathrm{e}-4$ \\
\hline $\mathrm{CV}$ & 0.204 & 0.201 & 0.204 & 0.204 \\
\hline & 7.19 e-5 & 6.14 e-5 & $6.73 \mathrm{e}-5$ & 5.74 e-5 \\
\hline $\mathrm{IS}+\mathrm{CV}$ & 0.204 & 0.204 & 0.204 & 0.204 \\
\hline & $3.61 \mathrm{e}-5$ & 2.90 e-5 & 3.12 e-5 & $3.08 \mathrm{e}-5$ \\
\hline & & Eur & eean & \\
\hline naive & 0.202 & 0.202 & 0.203 & 0.202 \\
\hline & $1.11 \mathrm{e}-3$ & $3.13 \mathrm{e}-4$ & $5.34 \mathrm{e}-4$ & 3.54 e-4 \\
\hline IS & 0.202 & 0.203 & 0.202 & 0.202 \\
\hline & $2.76 \mathrm{e}-4$ & $1.54 \mathrm{e}-4$ & 1.76 e-4 & $1.51 \mathrm{e}-4$ \\
\hline $\mathrm{CV}$ & 0.202 & 0.202 & 0.202 & 0.202 \\
\hline & 3.76 e-5 & $2.31 \mathrm{e}-5$ & 2.88 e- 5 & 2.35 e-5 \\
\hline $\mathrm{IS}+\mathrm{CV}$ & 0.202 & 0.202 & 0.202 & 0.202 \\
\hline & 2.09 e- 5 & $1.50 \mathrm{e}-5$ & 1.79 e-5 & $1.61 \mathrm{e}-5$ \\
\hline
\end{tabular}




\section{Lemieux and La}

Table 2: $T=1, \sigma=0.3$, and $b=64$

\begin{tabular}{|c|c|c|c|c|}
\hline & $\mathrm{MC}$ & Sobol' & Kor & PKor \\
\hline$K=45$ & \multicolumn{4}{|c|}{ American } \\
\hline \multirow[t]{2}{*}{ naive } & 8.243 & 8.223 & 8.218 & 8.221 \\
\hline & 9.96 e-3 & $2.75 \mathrm{e}-3$ & 2.79 e-3 & $2.85 \mathrm{e}-3$ \\
\hline \multirow[t]{2}{*}{ IS } & 8.077 & 8.079 & 8.074 & 8.073 \\
\hline & $4.23 \mathrm{e}-3$ & $2.23 \mathrm{e}-3$ & $2.67 \mathrm{e}-3$ & $2.33 \mathrm{e}-3$ \\
\hline \multirow[t]{2}{*}{$\mathrm{CV}$} & 8.227 & 8.224 & 8.218 & 8.222 \\
\hline & 3.80 e-3 & $2.65 \mathrm{e}-3$ & $2.68 \mathrm{e}-3$ & $2.82 \mathrm{e}-3$ \\
\hline \multirow[t]{3}{*}{$\mathrm{IS}+\mathrm{CV}$} & 8.079 & 8.079 & 8.074 & 8.074 \\
\hline & 3.49 e-3 & $2.19 \mathrm{e}-3$ & $2.50 \mathrm{e}-3$ & $2.18 \mathrm{e}-3$ \\
\hline & \multicolumn{4}{|c|}{ European } \\
\hline \multirow[t]{2}{*}{ naive } & 7.039 & 7.019 & 7.020 & 7.023 \\
\hline & $1.12 \mathrm{e}-2$ & $1.42 \mathrm{e}-3$ & 1.79 e-3 & $1.06 \mathrm{e}-3$ \\
\hline \multirow[t]{2}{*}{ IS } & 7.018 & 7.020 & 7.022 & 7.018 \\
\hline & $3.73 \mathrm{e}-3$ & $1.76 \mathrm{e}-3$ & $1.91 \mathrm{e}-3$ & $1.72 \mathrm{e}-3$ \\
\hline \multirow[t]{2}{*}{$\mathrm{CV}$} & 7.021 & 7.021 & 7.021 & 7.021 \\
\hline & $4.10 \mathrm{e}-4$ & $2.37 \mathrm{e}-4$ & $2.57 \mathrm{e}-4$ & $1.52 \mathrm{e}-4$ \\
\hline \multirow[t]{2}{*}{$\mathrm{IS}+\mathrm{CV}$} & 7.020 & 7.020 & 7.021 & 7.021 \\
\hline & $3.43 \mathrm{e}-4$ & $1.96 \mathrm{e}-4$ & $1.94 \mathrm{e}-4$ & $1.98 \mathrm{e}-4$ \\
\hline$K=50$ & \multicolumn{4}{|c|}{ American } \\
\hline \multirow[t]{2}{*}{ naive } & 4.507 & 4.492 & 4.491 & 4.492 \\
\hline & $9.31 \mathrm{e}-3$ & $2.50 \mathrm{e}-3$ & $2.18 \mathrm{e}-3$ & $1.76 \mathrm{e}-3$ \\
\hline \multirow[t]{2}{*}{ IS } & 4.454 & 4.454 & 4.451 & 4.451 \\
\hline & 3.38 e- 3 & $1.92 \mathrm{e}-3$ & $2.02 \mathrm{e}-3$ & $1.91 \mathrm{e}-3$ \\
\hline \multirow[t]{2}{*}{$\mathrm{CV}$} & 4.494 & 4.493 & 4.491 & 4.491 \\
\hline & $2.46 \mathrm{e}-3$ & $1.79 \mathrm{e}-3$ & $1.70 \mathrm{e}-3$ & $1.63 \mathrm{e}-3$ \\
\hline \multirow[t]{3}{*}{$\mathrm{IS}+\mathrm{CV}$} & 4.453 & 4.453 & 4.451 & 4.452 \\
\hline & $2.01 \mathrm{e}-3$ & $1.48 \mathrm{e}-3$ & $1.68 \mathrm{e}-3$ & $1.42 \mathrm{e}-3$ \\
\hline & \multicolumn{4}{|c|}{ European } \\
\hline \multirow[t]{2}{*}{ naive } & 4.036 & 4.022 & 4.023 & 4.024 \\
\hline & 9.47 e-3 & $1.82 \mathrm{e}-3$ & $2.09 \mathrm{e}-3$ & $1.51 \mathrm{e}-3$ \\
\hline \multirow[t]{2}{*}{ IS } & 4.023 & 4.023 & 4.022 & 4.021 \\
\hline & 3.09 e-3 & $1.65 \mathrm{e}-3$ & $1.77 \mathrm{e}-3$ & $1.78 \mathrm{e}-3$ \\
\hline \multirow[t]{2}{*}{$\mathrm{CV}$} & 4.022 & 4.023 & 4.023 & 4.022 \\
\hline & 3.77 e-4 & $2.22 \mathrm{e}-4$ & $2.47 \mathrm{e}-4$ & $1.54 \mathrm{e}-4$ \\
\hline $\mathrm{IS}+\mathrm{CV}$ & 4.022 & 4.022 & 4.022 & 4.022 \\
\hline & $2.51 \mathrm{e}-4$ & $1.47 \mathrm{e}-4$ & $1.85 \mathrm{e}-4$ & $1.36 \mathrm{e}-4$ \\
\hline$K=55$ & & $\mathrm{Am}$ & ican & \\
\hline naive & 2.236 & 2.225 & 2.225 & 2.225 \\
\hline & $7.32 \mathrm{e}-3$ & $2.36 \mathrm{e}-3$ & $2.40 \mathrm{e}-3$ & $1.87 \mathrm{e}-3$ \\
\hline IS & 2.221 & 2.221 & 2.219 & 2.219 \\
\hline & $2.19 \mathrm{e}-3$ & $1.32 \mathrm{e}-3$ & $1.39 \mathrm{e}-3$ & $1.33 \mathrm{e}-3$ \\
\hline $\mathrm{CV}$ & 2.228 & 2.224 & 2.225 & 2.225 \\
\hline & $1.29 \mathrm{e}-3$ & $1.08 \mathrm{e}-3$ & $1.13 \mathrm{e}-3$ & $1.07 \mathrm{e}-3$ \\
\hline $\mathrm{IS}+\mathrm{CV}$ & 2.219 & 2.220 & 2.219 & 2.220 \\
\hline & $8.34 \mathrm{e}-4$ & 7.40 e- 4 & 7.63 e- 4 & $6.54 \mathrm{e}-4$ \\
\hline & & Eur & ean & \\
\hline naive & 2.088 & 2.082 & 2.079 & 2.079 \\
\hline & $1.20 \mathrm{e}-2$ & 2.14 e-3 & $2.46 \mathrm{e}-3$ & $1.73 \mathrm{e}-3$ \\
\hline IS & 2.081 & 2.080 & 2.080 & 2.079 \\
\hline & $2.06 \mathrm{e}-3$ & $1.28 \mathrm{e}-3$ & $1.34 \mathrm{e}-3$ & $1.28 \mathrm{e}-3$ \\
\hline $\mathrm{CV}$ & 2.079 & 2.080 & 2.080 & 2.080 \\
\hline & $3.63 \mathrm{e}-4$ & $1.98 \mathrm{e}-4$ & $2.63 \mathrm{e}-4$ & $1.78 \mathrm{e}-4$ \\
\hline $\mathrm{IS}+\mathrm{CV}$ & 2.079 & 2.080 & 2.080 & 2.080 \\
\hline & $2.30 \mathrm{e}-4$ & $1.39 \mathrm{e}-4$ & $1.75 \mathrm{e}-4$ & $1.53 \mathrm{e}-4$ \\
\hline
\end{tabular}

Table 3: $T=1, \sigma=0.3, b=64$, and $K=60$

\begin{tabular}{|l|l|r|r|r|}
\hline & MC & Sobol' & Kor & PKor \\
\hline & \multicolumn{4}{|c|}{ American } \\
\hline naive & 1.030 & 1.027 & 1.025 & 1.021 \\
& $5.39 \mathrm{e}-3$ & $2.13 \mathrm{e}-3$ & $2.57 \mathrm{e}-3$ & $1.95 \mathrm{e}-3$ \\
IS & 1.025 & 1.024 & 1.024 & 1.024 \\
& $1.25 \mathrm{e}-3$ & $8.23 \mathrm{e}-4$ & $8.31 \mathrm{e}-4$ & $8.04 \mathrm{e}-4$ \\
CV & 1.025 & 1.025 & 1.023 & 1.024 \\
& $7.29 \mathrm{e}-4$ & $5.41 \mathrm{e}-4$ & $6.54 \mathrm{e}-4$ & $5.60 \mathrm{e}-4$ \\
IS+CV & 1.024 & 1.024 & 1.024 & 1.024 \\
& $3.69 \mathrm{e}-4$ & $3.24 \mathrm{e}-4$ & $3.45 \mathrm{e}-4$ & $3.15 \mathrm{e}-4$ \\
\hline \multirow{4}{*}{ naive } & 0.989 & 0.987 & 0.986 & 0.981 \\
& $5.31 \mathrm{e}-3$ & $2.16 \mathrm{e}-3$ & $2.50 \mathrm{e}-3$ & $1.99 \mathrm{e}-3$ \\
IS & 0.985 & 0.984 & 0.985 & 0.984 \\
& $1.20 \mathrm{e}-3$ & $7.80 \mathrm{e}-4$ & $7.93 \mathrm{e}-4$ & $7.85 \mathrm{e}-4$ \\
CV & 0.984 & 0.985 & 0.985 & 0.984 \\
& $3.43 \mathrm{e}-4$ & $2.46 \mathrm{e}-4$ & $2.39 \mathrm{e}-4$ & $2.14 \mathrm{e}-4$ \\
IS+CV & 0.984 & 0.984 & 0.984 & 0.984 \\
& $1.63 \mathrm{e}-4$ & $1.40 \mathrm{e}-4$ & $1.70 \mathrm{e}-4$ & $1.34 \mathrm{e}-4$ \\
\hline
\end{tabular}

More generally, we can say that since both CV and IS are designed to work well for the European Asian option, we can expect them to work better for American option's with a smaller early exercise premium. The reduction factors obtained in this case are quite interesting: for instance the standard error for Monte Carlo is reduced by a factor of about 30 when $K=55$ in Table 1, when both IS anc CV are used. The reduction factors continue to increase as the option becomes more out-the-money, as seen in Table 3 , where the parameters are the same as in Table 2, but $K=60$.

Turning to the performance of RQMC compared to IS and/or CV, we see that Monte Carlo becomes competitive with RQMC when IS and CV are applied, especially for options that are out-of-the money (since as discussed above, those are the cases where IS and CV work better). However, RQMC estimators still outperform Monte Carlo in all cases. Also, we can compare the individual performance of RQMC with that of IS and $\mathrm{CV}$ taken alone: the reduction factors brought by RQMC alone (compare the second to fourth column to the first one for the naive row) vary between 2 and 8, which is not as good as the best performance seen with CV (factor of about 30, as discussed previously), but is better than what IS does, with the advantage that RQMC does not make the bias larger. Note also that applying RQMC does not require any extra work: in fact, simulations based on HUPS tend to be faster than Monte Carlo.

\section{CONCLUSION}

In this paper we investigated the effectiveness of different variance reduction techniques to improve estimators obtained in the Longstaff-Schwartz approach. We only 


\section{Lemieux and La}

looked at one type of option - an Asian call - and have seen that for this particular case, importance sampling and a control variate can help reducing the variance, but that their usefulness is sensitive to the choice of parameters for the option. Randomized quasi-Monte Carlo methods have been used as well, and have been seen to consistently reduce the variance with respect to Monte Carlo, whether other techniques are used or not.

For future research, it would obviously be important to extend our investigations to other types of options. We expect that in some cases, schemes other than the one used here for importance sampling will need to be used. A promising approach is the one discussed in Bolia, Glasserman, and Juneja (2004).

\section{ACKNOWLEDGMENTS}

This work was supported by NSERC grant \#RGP238959. We thank Anatoliy Swischuck for his helpful comments.

\section{REFERENCES}

Avramidis, A. N., and P. Hyden. 1999. Efficiency improvements for pricing American options with a stochastic mesh. In Proceedings of the 1999 Winter Simulation Conference, ed. P. A. Farrington, H. B. Nembhard, D. T. Sturrock, and G. W. Evans, 344-350. Piscataway, New Jersey: IEEE Press.

Bolia, N., P. Glasserman, and S. Juneja. 2004. Functionapproximation-based importance sampling for pricing American options. In Proceedings of the 2004 Winter Simulation Conference, ed. R. G. Ingalls, M. D. Rossetti, J. S. Smith, and B. A. Peters, 604-611. Piscataway, New Jersey: IEEE Press.

Boyle, P., A. W. Kolkiewicz, and K. S. Tan. 2003. Am improved simulation method for pricing high-dimensional American derivatives. Mathematics and Computers in Simulation 62:315-322.

Bratley, P., and B. L. Fox. 1988. Algorithm 659: Implementing Sobol's quasirandom sequence generator. $A C M$ Transactions on Mathematical Software 14 (1): 88-100.

Broadie, M., and P. Glasserman. 1997. A stochastic mesh method for pricing high-dimensional American options. Manuscript.

Chaudhary, S. K. 2004. Acceleration of Monte Carlo methods using low discrepancy sequences. Ph. D. thesis, Department of Mathematics, University of California, Los Angeles.

Duffie, D. 1996. Dynamic asset pricing theory. second ed. Princeton University Press.

Glasserman, P. 2004. Monte Carlo methods in financial engineering, Volume 53 of Application of Mathematics - Stochastic Modelling and Applied Probability. New York: Springer.
Glasserman, P., P. Heidelberger, and P. Shahabuddin. 1999. Asymptotically optimal importance sampling and stratification for pricing path dependent options. Journal of Mathematical Finance 9 (2): 117-152.

Haugh, M., and L. Kogan. 2004. Pricing American options: a duality approach. Operations Research 52:258-270.

Hickernell, F. J., C. Lemieux, and A. B. Owen. 2005. Control variates for quasi-Monte Carlo. Statistical Science 20:131.

Kemna, A. G. Z., and A. C. F. Vorst. 1990. A pricing method for options based on average asset values. Journal of Banking and Finance 14:113-129.

Korobov, N. M. 1959. The approximate computation of multiple integrals. Dokl. Akad. Nauk SSSR 124:12071210. In Russian.

L'Ecuyer, P. 2004. Quasi-Monte Carlo methods in finance. In Proceedings of the 2004 Winter Simulation Conference, ed. R. G. Ingalls, M. D. Rossetti, J. S. Smith, and B. A. Peters, 1645-1655. Piscataway, New Jersey: IEEE Press.

L'Ecuyer, P., and C. Lemieux. 2002. Recent advances in randomized quasi-Monte Carlo methods. In Modeling Uncertainty: An Examination of Stochastic Theory, Methods, and Applications, ed. M. Dror, P. L'Ecuyer, and F. Szidarovszki, 419-474: Kluwer Academic Publishers, Boston.

Lemieux, C. 2004. Randomized quasi-Monte Carlo methods: a tool for improving the efficiency of simulations in finance. In Proceedings of the 2004 Winter Simulation Conference, ed. R. G. Ingalls, M. D. Rossetti, J. S. Smith, and B. A. Peters, 1565-1573. Piscataway, New Jersey: IEEE Press.

Lemieux, C., and P. L'Ecuyer. 2003. Randomized polynomial lattice rules for multivariate integration and simulation. SIAM J. Sci. Comp. 24 (5): 1768-1789.

Longstaff, F. A., and E. S. Schwartz. 2001. Valuing American options by simulations: A simple least-squares approach. Review of Financial Studies 14 (1): 113147.

Moreni, N. 2003. Pricing American options: A variance reduction technique for the Longstaff-Schwartz algorithm. Technical Report 2003-256, CERMICS-ENPC, Champs-sur-Marne.

Owen, A. B. 1998. Latin supercube sampling for very highdimensional simulations. ACM Trans. Model. Comp. Simul. 8 (1): 71-102.

Sobol', I. M. 1967. The distribution of points in a cube and the approximate evaluation of integrals. U.S.S.R. Comput. Math. and Math. Phys. 7:86-112.

$\mathrm{Su}, \mathrm{Y}$., and M. Fu. 2000. Importance sampling in derivative securities pricing. In Proceedings of the 2000 Winter Simulation Conference, 587-596. Piscataway, NJ: IEEE Press. 


\section{Lemieux and La}

Vázquez-Abad, F., and D. Dufresne. 1998. Accelerated simulation for pricing Asian options. In Proceedings of the 1998 Winter Simulation Conference, 1493-1500: IEEE Press.

\section{AUTHOR BIOGRAPHIES}

CHRISTIANE LEMIEUX is an associate professor at the University of Calgary in the departments of mathematics and statistics, and computer science. She has a Ph.D. in Computer Science from the University of Montreal. She is a member of INFORMS Simulation Society and an associate of the Society of Actuaries. Her e-mail address is <lemieux@math.ucalgary.cas and her Web address is <http: //www. math.ucalgary.ca/ $\sim$ lemieux $>$.

JENNIE LA is a masters student in statistics at the University of Calgary. She has a B.Sc. in Microbiology from the University of Manitoba and a B.Sc. in Actuarial Science from the University of Calgary. Her e-mail address is $<$ laj@math. ucalgary.ca>. 